\title{
COVID-19: what happened to all of the otolaryngology emergencies?
}

\author{
Matteo Gelardi ${ }^{1}$ - Lucia lannuzzi ${ }^{2}$ Eleonora M. C. Trecca ${ }^{1}$ (D - Brandon Kim ${ }^{3}$ - Nicola A. A. Quaranta ${ }^{2}$. \\ Michele Cassano ${ }^{1}$
}

Received: 14 April 2020 / Accepted: 10 May 2020 / Published online: 18 May 2020

(c) Springer-Verlag GmbH Germany, part of Springer Nature 2020

Keywords Emergency medicine $\cdot$ Critical care $\cdot$ Hospital administration $\cdot$ Ambulatory care $\cdot$ Primary health care . Otolaryngology $\cdot$ COVID-19

To the Editor,

COVID-19 is a global pandemic caused by the novel coronavirus SARS-CoV-2, and Italy was the first European country to experience a large-scale outbreak. With this, every aspect of healthcare, the economy, and society has been deeply affected in Italy, so much so that fundamental changes are expected in the post-COVID-19 era. One aspect is that access to emergency care in Otolaryngology (ORL) patients has already changed in the month following the onset of the COVID-19 outbreak, and, seemingly, many non-COVID illnesses have been disappearing.

Although the government did not enact any limitations or prohibitions to emergency access for patients afflicted by acute ORL pathologies, the number of daily urgent consultations to the ORL Departments in Bari and Foggia University Hospitals has significantly reduced and, on some days, was even zero.

One possible reason for this change may be patients' fear of acquiring a nosocomial COVID-19 infection. Even so, it is difficult to believe that some acute diseases, such as massive epistaxis, otitis, or pharyngotonsillitis, could have occurred so rarely or not at all or, alternately, could have been treated at home by patients or their families. Rather, most certainly, primary care physicians, who have been

Eleonora M. C. Trecca

eleonoramc.trecca@gmail.com; eleonora.trecca@unifg.it

1 Department of Otolaryngology-Head and Neck Surgery, University Hospital of Foggia, Viale Pinto, 1, 71121 Foggia, Italy

2 Department of Otolaryngology-Head and Neck Surgery, University of Bari ‘Aldo Moro', Bari, Italy

3 Department of Otolaryngology-Head and Neck Surgery, The Ohio State University Wexner Medical Center, Columbus, $\mathrm{OH}, \mathrm{USA}$ performing telehealth consultations in accordance with the guidelines from the Higher Institute of Health (ISS), have been providing an invaluable service [1]; it is posited that patients who received primary care services this way may have been able to delay or even forego specialist evaluation. This telehealth treatment has not only helped to keep patients at home but has provided other benefits and should be considered in the future.

Table 1 shows data regarding the number of emergency department consultations by diagnosis in the 30 days preceding (from February 8th to March 8th, 2020) and 30 days following (from March 9th to April 9th, 2020) the onset of the COVID-19 outbreak at the two main tertiary referral centers in the Puglia region, as well as the percentage reduction for each diagnosis.

The data revealed an overall decrease in the number of ORL emergency consultations by $80.8 \%$. Of note, reductions were noted across all subspecialties.

Although some illnesses, such as rhinitis, sinusitis, uvular edema, and tinnitus, do not usually require immediate attention, it is more difficult to explain the large reduction, ranging from $80.2 \%$ to $88.9 \%$, of other diagnoses, such as vertigo, epistaxis, sudden hearing loss, otitis media, and peritonsillar abscesses, which often merit urgent ORL consultations. The reduction of some diagnoses may be the consequence of national lockdown orders. For example, the $84.6 \%$ reduction in the number of nasal bone fractures may be related to the near-absence of motor vehicle accidents reported during this time. In contrast, it is more difficult to explain the reduction of other acute diseases such as sudden hearing loss, whose frequency decreased $83.3 \%$, especially when compared to non-disease conditions, such as cerumen impaction, whose reduction in presentation, interestingly, was slightly more modest at $73.7 \%$. 
Table 1 Otolaryngology (ORL) emergencies before and after the onset of the COVID-19 outbreak

\begin{tabular}{lccl}
\hline Diseases & $\begin{array}{l}\text { Pre- } \\
\text { COVID-19 } \\
\text { time* }\end{array}$ & $\begin{array}{l}\text { Post- } \\
\text { COVID-19 } \\
\text { time** }\end{array}$ & $\begin{array}{l}\text { Percentage } \\
\text { decreases } \\
(\%)\end{array}$ \\
\hline Vertigo & 125 & 15 & -88 \\
Otitis media & 88 & 12 & -86.4 \\
Epistaxis & 86 & 17 & -80.2 \\
Pharyngotonsillitis & 69 & 17 & -75.4 \\
Cerumen impaction & 38 & 10 & -73.7 \\
Foreign bodies & 27 & 7 & -74.1 \\
Nasal bone fractures & 26 & 4 & -84.6 \\
Facial trauma & 21 & 6 & -71.4 \\
Otitis externa & 18 & 9 & -50 \\
Tinnitus & 16 & 0 & -100 \\
Sinusitis & 14 & 0 & -100 \\
Sudden hearing loss & 12 & 2 & -83.3 \\
Uvular edema & 11 & 0 & -100 \\
Rhinitis & 11 & 0 & -100 \\
Peritonsillar abscess & 9 & 1 & -88.9 \\
Laryngitis & 7 & 5 & -28.6 \\
Miscellanea & 42 & 11 & -73.8 \\
Absence of ENT disease & 30 & 9 & -70 \\
Total & 650 & 125 & -80.8 \\
\hline
\end{tabular}

*Pre-COVID-19 time: from February 8th to March 8th, 2020. **PostCOVID-19 time: from March 9th to April 9th, 2020

A reduction in the number of emergency ORL consultations by $80.8 \%$ during the COVID-19 pandemic creates new paradigms for the future. Over the past few decades, the workload performed by healthcare personnel in emergency departments has increased, and some have even noted a reduction in morale and job satisfaction, as their responsibilities include intervening in not only true emergencies but also managing many patients with non-acute complaints. One possible exacerbating factor is the length of waitlists to access outpatient care in the Italian National Healthcare System (NHS), as some patients may have to wait for months to see an otolaryngologist. This situation has motivated many to seek care in the emergency department to skip wait times and receive specialty evaluation and treatment without charge.
Decreasing the utilization of emergency department services by improving access to primary care services has been a long-term aim, articulated since at least 1958, when physicians working at Hartford Hospital published a pioneering piece in The New England Journal of Medicine [2] that showed that only a minimum number of patients who visited the emergency department required inpatient care. At that time, proposed solutions were to increase utilization of outpatient care and to educate patients to seek a preliminary triage consultation with their family physicians; it is possible that the COVID-19 outbreak is pushing our current society in a similar direction.

Certainly, the priority now is to fight the pandemic, but in time, many aspects of healthcare should be reviewed and better organization through telemedicine sought [3]. Hopefully, patients will have learnt to more appropriately utilize the numerous resources offered by the Italian NHS for all of its patients.

\section{Funding None.}

\section{Compliance with ethical standards}

Conflict of interest The authors disclose no conflicts of interest.

\section{References}

1. Basu S, Phillips RS (2016) Reduced emergency department utilization after increased access to primary care. PLOS Med 13(9):e1002114. https://doi.org/10.1371/journal.pmed.1002114

2. Shortliffe EC, Hamilton TS, Noroian EH (1958) The emergency room and the changing pattern of medical care. N Engl J Med 258(1):20-25. https://doi.org/10.1056/NEJM195801022580105

3. Bhuyan SS, Saha A, Bhatt J (2016) Reducing emergency department use requires interventions at multiple levels. Am J Emerg Med 34(6):1175-1176. https://doi.org/10.1016/j. ajem.2016.03.057

Publisher's Note Springer Nature remains neutral with regard to jurisdictional claims in published maps and institutional affiliations. 